ST/F-TN-08/11

\title{
Beam energy spread in FERMI@elettra gun and linac induced by intrabeam scattering
}

Alexander A. Zholents and Max S. Zolotorev

Lawrence Berkeley National Laboratory, University of California,

Berkeley, California 94720

Giuseppe Penco

Sincrotrone Trieste, Italy

June 2008

\section{Disclaimer}

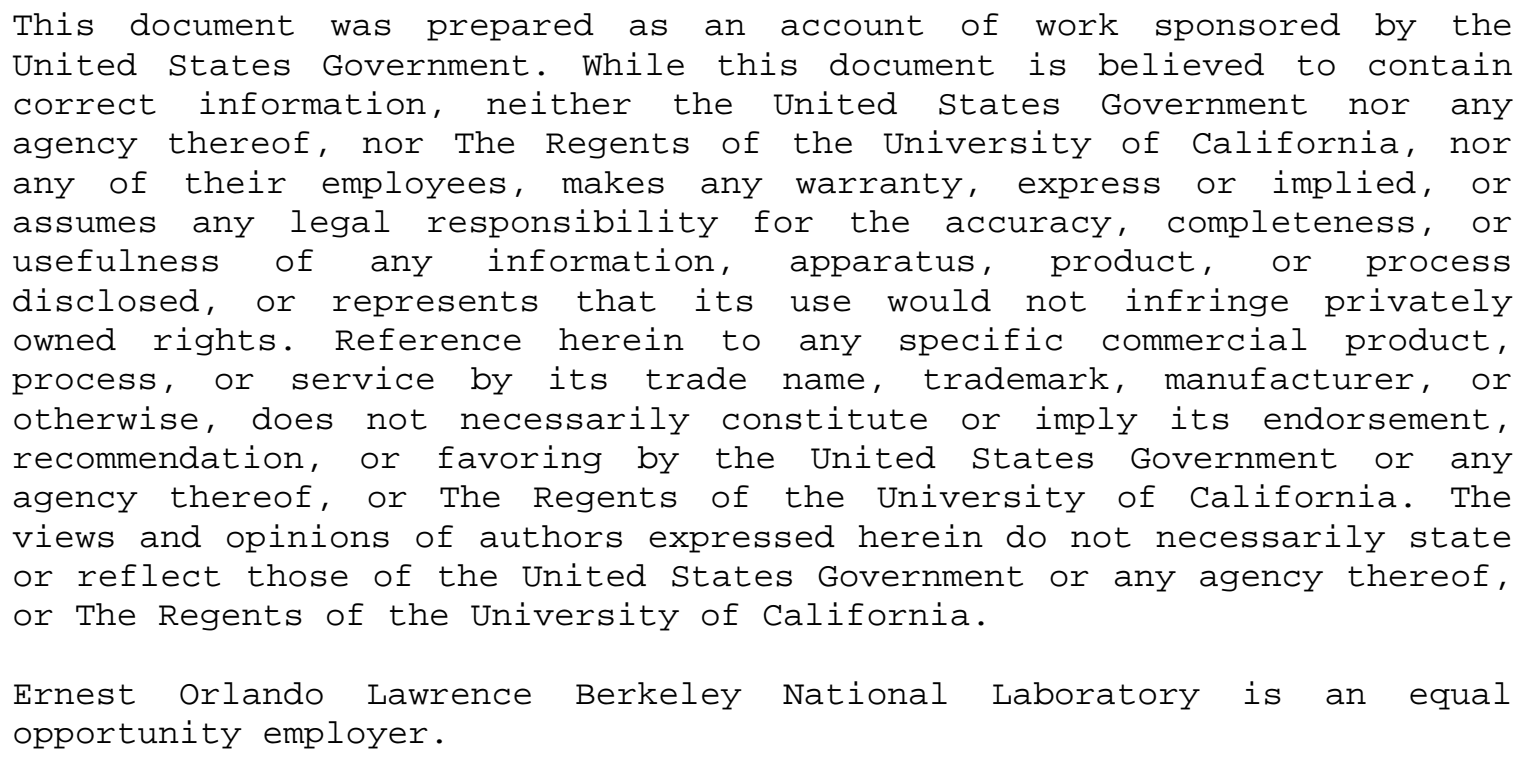




\section{Introduction}

Intrabeam scattering (IBS) of electrons in the pre-cathode area in the electron guns know in the literature as Boersh effect (see [1] and also textbook [2]) is responsible for a growth of the electron beam energy spread there. Albeit most visible within the electron gun where the electron beam density is large and the energy spread is small, the IBS acts all along the entire electron beam pass through the Linac. In this Note we calculate the energy spread induced by IBS in the FERMI@elettra gun [3,4] and also estimate its further growth through the linac up to the Laser Heater area. In our calculations we used actual beam sizes obtained via particle tracking with GPT code [5] for the part of the gun beginning right next to the cathode and up to approximately $5 \mathrm{MeV}$ beam energy and applied Piwinski formalism [6] to obtain energy spread induced by IBS. In the rest analysis performed for the electron beam pass from $5 \mathrm{MeV}$ and up to $100 \mathrm{MeV}$ we used constant beta-function of $5 \mathrm{~m}$ and applied simplified expression derived from [6].

\section{Results}

Figure 1 shows the evolution of the electron beam energy in the FERMI@elettra electron gun beginning from the cathode. Figure 2 and 3 show the evolution of the rms horizontal and vertical beam size $\sigma_{x, y}$ and rms horizontal and vertical beam divergence $\sigma_{x^{\prime}, y^{\prime}}$. Figure 4 show the evolution of the rms bunch length $\sigma_{z}$. In our case we deal with a cylindrical beam with equal horizontal and vertical sizes and Gaussian transverse distribution. We also assume Gaussian longitudinal distribution with the same rms beam size equal to the rms size obtained in simulations for distribution which is closer to a parabolic distribution [3]. Assuming bunch charge of $0.8 \mathrm{nC}$ and carrying out calculations beginning from approximately $3 \mathrm{~mm}$ after the cathode and using formalism [6], we obtain approximately $\sigma_{e}=55 \mathrm{eV}$ for the rms electron beam energy spread induced by IBS.

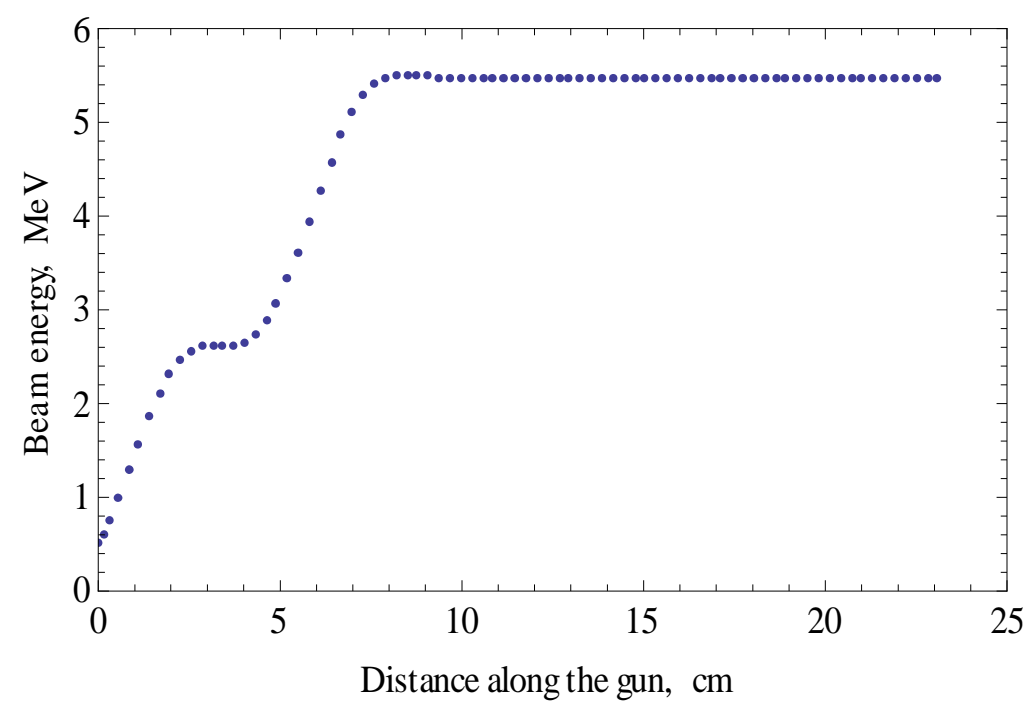

Figure 1. The evolution of the electron beam energy along the electron gun. 


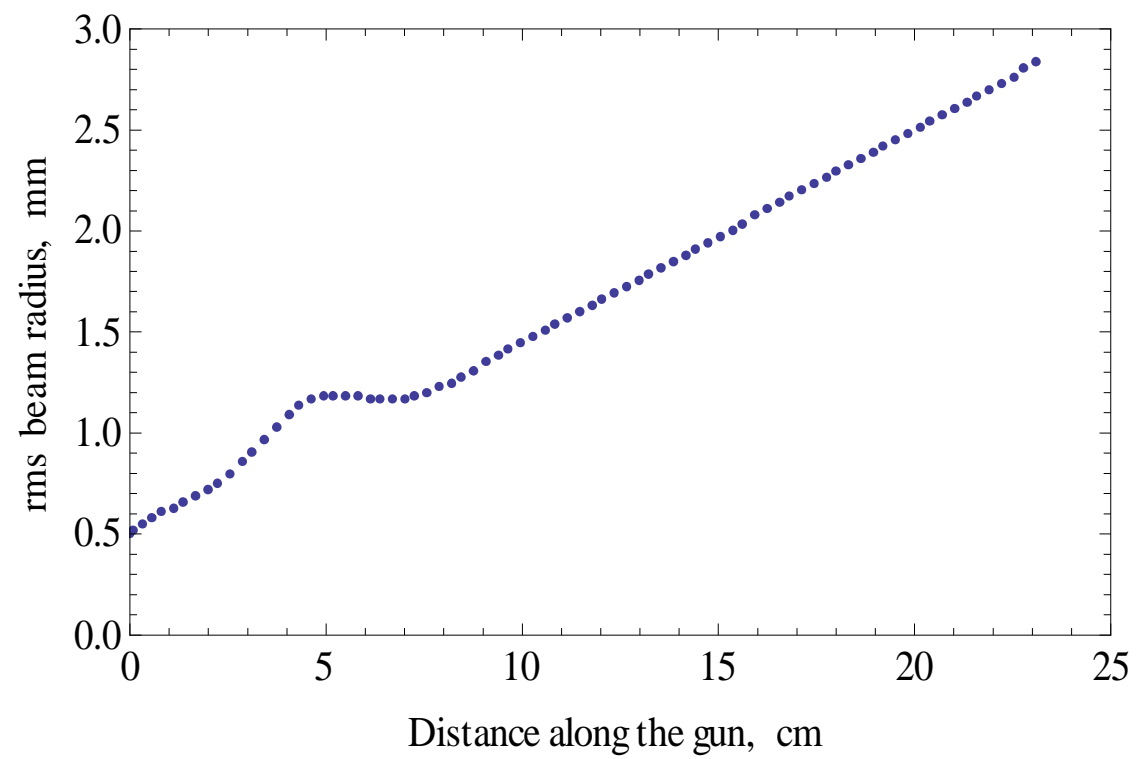

Figure 2. RMS beam horizontal and vertical size at various locations along the electron gun.

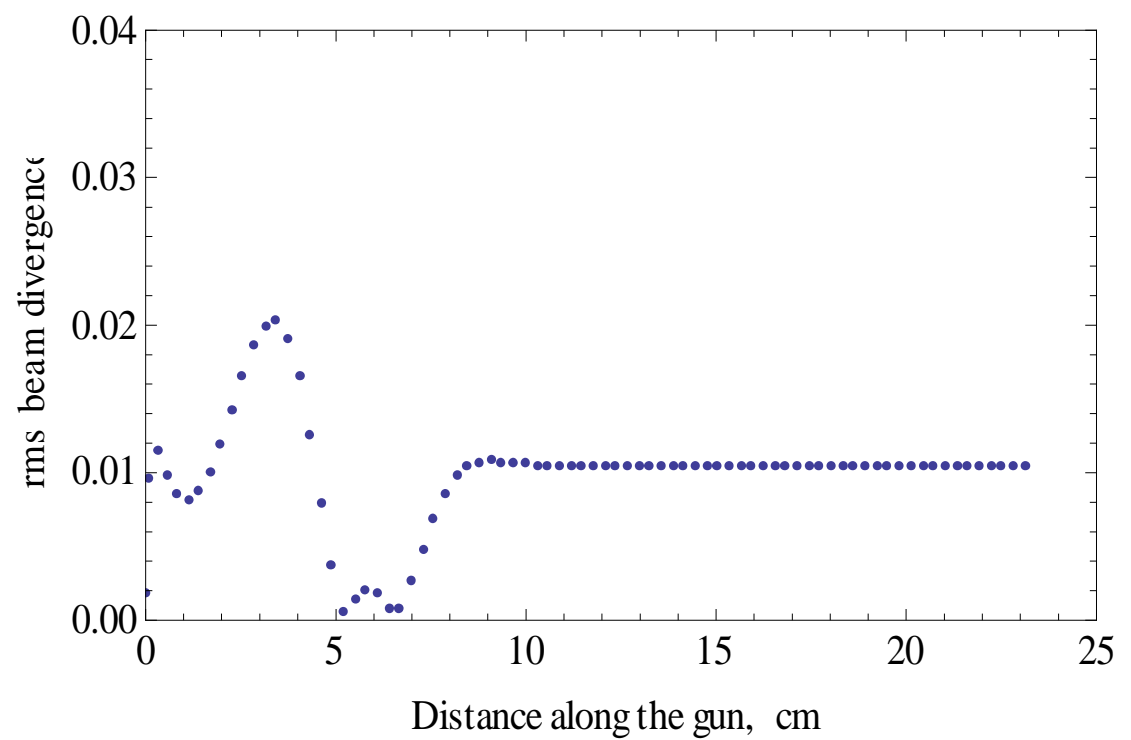

Figure 3. RMS beam horizontal and vertical divergence at various locations along the electron gun. 


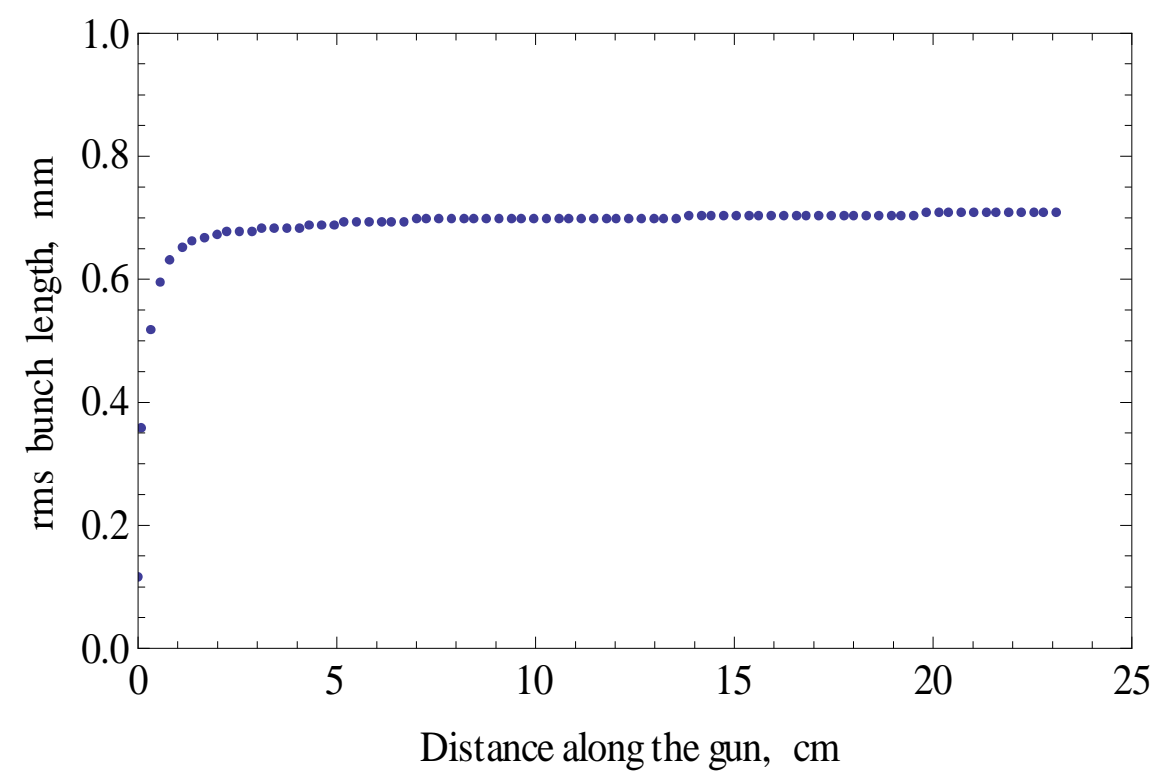

Figure 4. RMS bunch length at various locations along the electron gun.

For the rest of the analysis we use the following expression derived from [6] to estimate the energy spread growth induced by IBS:

$$
\frac{d \sigma_{e}^{2}}{d z}=\frac{r_{e}^{2} N \Lambda \gamma^{1 / 2}}{8 \sigma_{z} \varepsilon_{\perp}^{3 / 2} \beta^{1 / 2}}
$$

Here $r_{e}$ is the classical electron radius, $N$ is the number of electrons in the bunch, $\varepsilon_{\perp}$ is the normalized transverse emittance (cylindrical beam with equal horizontal and vertical emittance and equal horizontal and vertical beam sizes is assumed), $\beta$ is the beta-function, $\gamma$ is the Lorentz factor, and $\Lambda \approx 14$ is Coulomb logarithm. We also assume that the electron beam gains energy continuously with the energy gain of $10 \mathrm{MeV} / \mathrm{m}$ up to total energy of $100 \mathrm{MeV}$ (the final point in our analysis) and that the betafunction remains constant over the length of the accelerator and equal to $5 \mathrm{~m}$. Other parameters are: $\sigma_{z}=$ $0.7 \mathrm{~mm}$ and $\varepsilon_{\perp}=1.5 \mathrm{~mm}$-mrad. Figure 5 shows the growth of the energy spread induced by IBS beginning from $\sigma_{e}=55 \mathrm{eV}$. The energy spread at the last point where beam energy is $100 \mathrm{MeV}$ is approximately $750 \mathrm{eV}$. According to a design of FERMI@elettra linac [7], at this point the electron beam enters the Laser Heater. 


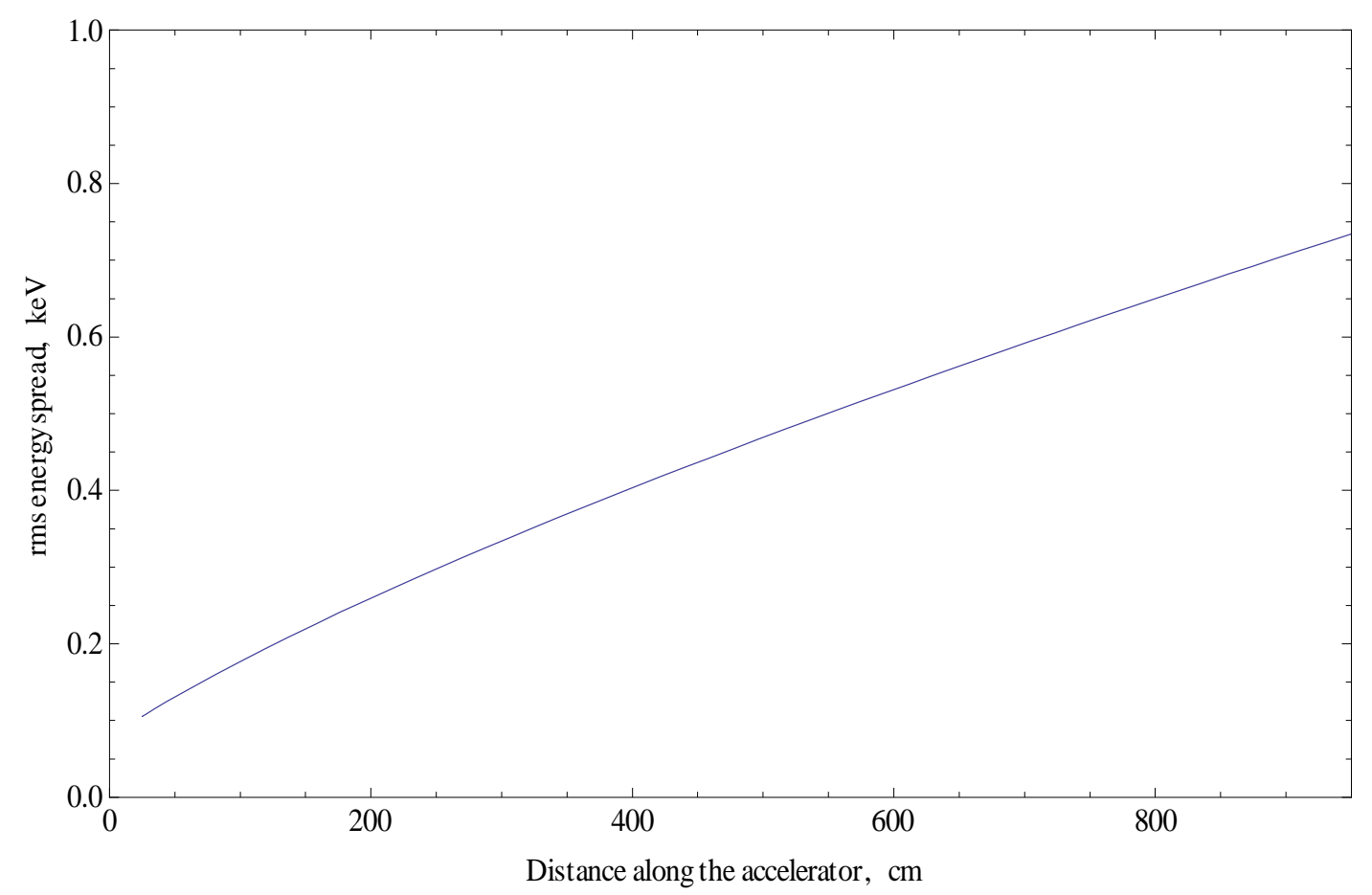

Figure 5. RMS energy spread induced by IBS at various locations along the accelerator.

Acknowledgement. Work supported by Department of Energy Contract No. DE-AC02$05 \mathrm{CH} 11231$.

\section{References:}

[1] H. Boersh, Z. Phys. , 139, 115(1954).

[2] M. Reiser, "Theory and Design of Charged Particle Beams", Wiley-VCH, Second edition, (2008).

[3] M. Trovò et al., "Status of the FERMI@Elettra Photoinjector", Proc of the EPAC 2008 conference.

[4] G.Penco, M.Trovo' and S.Lidia, “Optimization studies of the FERMI@ELETTRA photoinjector”, Proc of the EPAC 2006 conference.

[5] S.B. Van der Geer et al., "General Particle tracer Overview", http://www.pulsar.nl/gpt/index/html.

[6] A. Chao and M. Tigner, "Handbook of Accelerator Physics and Engineering", World Scientific, (1998)

p.126; see also A. Piwinski, CERN Accelerator School Advanced Accelerator Physics, CERN 87-03, p.402 (1987).

[7] Conceptual Design Report (CDR) for the FERMI@Elettra project, Sincrotrone Trieste, ST/F-TN-07/12 (2007). 